\title{
EVALUACIÓN DE LA UNIFORMIDAD DE DISTRIBUCIÓN EN CAMPO EN RIEGO POR GOTEO CON AGUAS REGENERADAS: COMPARACIÓN Y PROPUESTA DE UN NUEVO MÉTODO
}

\author{
Duran-Ros, M. (1); Arbat, G. (2); Pujol, J. (3); Barragán, J (4).; Ramírez de Cartagena, F. (5) \\ (P); Puig-Bargués, J. (6)
}

\begin{abstract}
1 Profesor Lector, Universidad de Girona, Departamento de Ingeniería Química, Agraria y Tecnología Agroalimentaria, miquel.duranros@udg.edu

2 Profesor Lector, Universidad de Girona, Departamento de Ingeniería Química, Agraria y Tecnología Agroalimentaria, gerard.arbat@udg.edu

3 Profesor Colaborador Permanente, Universidad de Girona, Departamento de Ingeniería Química, Agraria y Tecnología Agroalimentaria, joan.pujol@udg.edu

${ }^{4}$ Catedrático Emérito, Universidad de Lleida, Departamento de Ingeniería Agroforestal, barragan@eagrof.udl.es

5 Profesor Titular de Universidad, Universidad de Girona, Departamento de Ingeniería Química, Agraria y Tecnología Agroalimentaria, francisco.ramirez@udg.edu

6 Profesor Titular de Universidad, Universidad de Girona, Departamento de Ingeniería Química, Agraria y Tecnología Agroalimentaria, jaume.puig@udg.edu
\end{abstract}

\section{Resumen}

La uniformidad de distribución de caudales es una medida de evaluación de los sistemas de riego por goteo. Existen distintos métodos de evaluación de campo descritos para su determinación, entre los cuales destacan los de Merriam y Keller (1978), ASAE (1998) y Burt (2004). La principal diferencia entre estos métodos está en la localización y cantidad de emisores que se utilizan para el cálculo de la uniformidad. En la situación actual de aumento de la utilización de tecnologías de precisión, se propone una alternativa a los métodos existentes consistente en la utilización de registros de caudal y presión proporcionados por distintos medidores instalados en la subunidad de riego, permitiendo estimar la uniformidad de distribución de caudales en tiempo real y sin necesidad de utilización de mano de obra para esta laboriosa tarea. La nueva metodología permitiría, además, evaluar instalaciones de goteo enterrado que de otra manera sería complicado de medir frecuentemente. La evolución de los resultados obtenidos en función de las horas de riego con el método presentado es más parecida a la que se consigue con el método de Burt (2004) que a la obtenida con el método de Merriam y Keller (1978).

\section{Abstract}

Flow distribution uniformity is a parameter used for micro-irrigation evaluation. Different methods describe how to collect data and to calculate the distribution uniformity, such as the procedures developed by Merriam and Keller (1978), ASAE (1998) and Burt (2004). The main difference between these methods is the number and location of the drip emitters used for calculating the distribution uniformity. Nowadays, with increasing use of precision technologies, an alternative method is proposed to estimate distribution uniformity in real time and without using the manpower required by this time-consuming task. This method consists in using flow and pressure data collected by the devices installed in the drip irrigation unit. The proposed procedure will also allow to evaluate subsurface drip irrigation 
systems, which distribution uniformity otherwise would be very difficult to determine frequently. The evolution of the results obtained by the proposed method according to the irrigation time is more similar with that obtained by Burt's (2004) method than that obtained following Merriam and Keller's (1978) method.

\section{1- Introducción. Objetivo del trabajo}

La uniformidad de distribución de caudales $\left(U D_{q}\right)$ es uno de los criterios más utilizados para el diseño y evaluación de instalaciones de riego por goteo (Barragán et al., 2006). Cuando este parámetro se utiliza para la evaluación, permite conocer si existen diferencias importantes en la aplicación del agua al cultivo y determinar las causas que las provocan para, de esta forma, poder reducirlas y poder mantener el funcionamiento de la instalación tan cerca como sea posible de las condiciones establecidas en el diseño original (Wu et al., 2007)

La $U D_{q}$, que depende principalmente del diseño hidráulico, el coeficiente de variación de fabricación y la obturación de los emisores (Barragán et al., 2006), es un buen indicador de la obturación cuando se utiliza en la evaluación de los sistemas de riego por goteo siempre que en la instalación evaluada el diseño hidráulico sea el adecuado y el coeficiente de variación de fabricación de los goteros sea muy bajo. De esta forma, evaluar la $U_{\mathrm{q}}$ es especialmente interesante cuando se utilizan aguas de riego con un potencial elevado de obturación, como es el caso de las aguas residuales regeneradas. Cabe comentar que el uso de aguas regeneradas en la agricultura es una alternativa viable en zonas donde ésta es escasa o hay competencia por el uso y que, el riego por goteo es un sistema de riego adecuado desde el punto de visto de salud pública y medioambiental (Bucks et al., 1979).

Entre los métodos existentes para la determinación de la $U D_{q}$ en campo destacan los de Merriam y Keller (1978), adaptado por Vermeiren y Jobling (1986), ASAE (1998) o el de Burt (2004). Las diferencias entre ellos estriban básicamente en el número y localización de los goteros que se utilizan para determinar su descarga y presión de trabajo. Los dos métodos merecen comentarios críticos, estando entre los más destacados la idoneidad en la localización de los emisores que se muestrean en campo en el método de Merriam y Keller (1978) para la obtención de la media del caudal emitido de todos los goteros de la unidad y de su varianza (Juana et al., 2007). Es también un inconveniente de estos métodos de evaluación el hecho que ésta se realiza en la parcela y representa unos costos de mano de obra por tratarse de una tarea laboriosa.

En el presente trabajo se propone un nuevo método que facilita la determinación de la $U D_{q}$, y se comparan los resultados obtenidos con los procedentes de la aplicación de los métodos de Merriam y Keller (1978) y de Burt (2004). El método propuesto permite monitorizar la $U D_{q}$ sin la necesidad de realizar el muestro en campo.

\section{2- Materiales y métodos}

Los experimentos se realizaron con una subunidad de riego por goteo en superficie que tenía 4 laterales de $87 \mathrm{~m}$ de longitud y goteros autocompensantes integrados de 1,6 I/h con coeficiente de variación de fabricación inferior al $3 \%$ situados cada $30 \mathrm{~cm}$. La subunidad disponía de un cabezal de filtración que permitió operar con filtros de anillas, malla y arena, sucesivamente. La subunidad utilizaba como agua de riego el efluente suministrado por la Estación Depuradora de Aguas Residuales (EDAR) de Celrà (Girona). El ensayo duró 4500 $\mathrm{h}$ y los laterales se reemplazaron por otros nuevos cada $2000 \mathrm{~h}$ de funcionamiento. 
Se realizaron 13 evaluaciones del sistema de riego que requirieron la recogida manual de datos de caudales y presiones en la parcela de riego. Estas determinaciones se realizaron aproximadamente cada $500 \mathrm{~h}$ de riego y siguieron los métodos de Merriam y Keller (1978) y de Burt (2004) El método de la ASAE no se utilizó puesto que requiere aleatoriedad en la elección de los goteros y, precisamente, se quería utilizar siempre los mismos goteros en las evaluaciones para poder analizar con detalle el efecto de su posible obturación. Con el método de Merriam y Keller (1978), adaptado por Vermeiren y Jobling (1986), se determinó el caudal en 2 goteros contiguos localizados al inicio, a 1/3, a 2/3 de la longitud total y al final de los cuatro laterales, adoptando como caudal representativo de cada localización el caudal medio de los 2 goteros. El método de Burt (2004) requirió determinar los caudales en 4 goteros adyacentes del inicio del cada lateral, otros 4 goteros contiguos localizados en la mitad de los laterales y en los últimos 7 emisores de cada lateral (ver Figura 1).

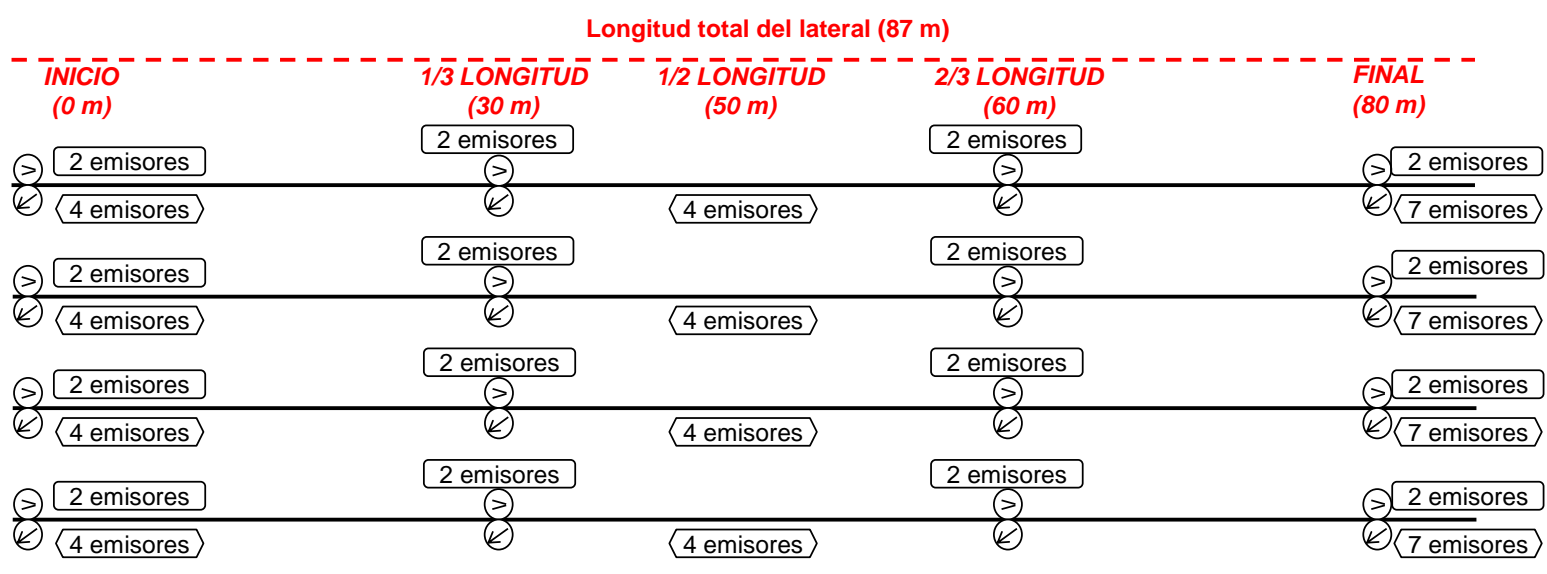

Leyenda:
$\ominus$ Medidor volumétrico (4) Transductor de presión
Emisor seleccionado para la determinación de la UD ${ }_{\mathrm{q}}$ por el método Merriam y Keller (1978)
Emisor seleccionado para la determinación de la $U_{\mathrm{q}}$ por el método Burt (2004)

Figura 1. Localización de los goteros según el método de evaluación de campo y localización de los medidores en continuo de volumen y presión.

La situación exacta desde la tubería terciaria de los medidores de volumen se observa en rojo en la Figura 1. Se decidió colocar el contador volumétrico del final del lateral a 7 metros del mismo en lugar del extremo final, para garantizar que el volumen medido fuera suficientemente alto para entrar dentro del rango de lectura del equipo.

Con los dos métodos de evaluación con recogida de datos manual, se midió la presión de trabajo de los goteros utilizando una toma de presión (Ein-Tal, Or-Akiva, Israel) y un manómetro digital modelo Leo 2 (Keller, Wintertur, Suiza) con un adaptador de aguja. Este manómetro tenía un error máximo de $0.065 \%$ de la lectura según el certificado de calibración emitido por el fabricante. El caudal de los emisores se determinó recogiendo el volumen emitido por cada uno de ellos durante 5 minutos en un recipiente de plástico de 5 $\mathrm{cm}$ de alto y $20 \mathrm{~cm}$ de diámetro (Figura 2). El volumen recogido estuvo comprendido entre 100 y $250 \mathrm{ml}$ tal y como se recomienda en el método de Merriam y Keller (1978). 


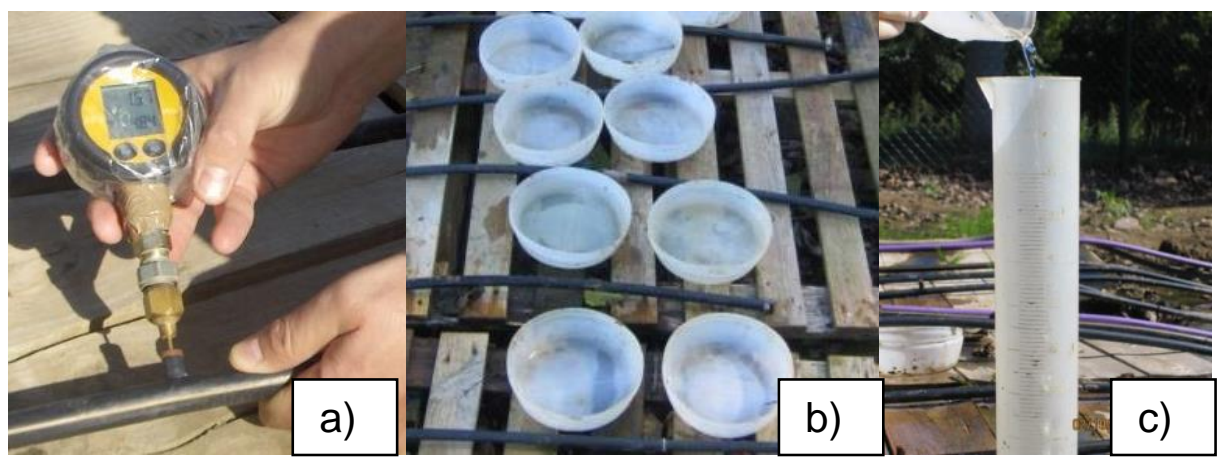

Figura 2. Manómetro digital con adaptador de aguja y toma de presión en un lateral de goteo (a) y proceso de recogida del volumen emitido por los emisores en las pruebas de uniformidad de distribución de caudales (b y c).

Con todos los datos de las evaluaciones de campo, se calculó la $U D_{q}$ y la uniformidad de distribución de presiones $\left(U D_{p}\right)$ con las ecuaciones (1) y (2), respectivamente, descritas en el método de Merriam y Keller (1978):

$$
U D_{q}=\frac{q_{25}}{\bar{q}} \cdot 100
$$

donde: $\quad \quad \quad \quad \mathrm{VD}_{\mathrm{q}}$ es la uniformidad de distribución de caudales (\%) $\mathrm{q}_{25}$ es el caudal medio del $25 \%$ de las localizaciones con un menor caudal $(\mathrm{l} / \mathrm{h})$, por lo que es la media de las 4 localizaciones con caudal representativo más bajo $\overline{\mathrm{q}}$ es el caudal medio de todas las posiciones muestreadas $(\mathrm{l} / \mathrm{h})$, por lo tanto, es la media de los 16 caudales representativos

$$
U D_{p}=\left(\frac{p_{25}}{\bar{p}}\right)^{x} \cdot 100
$$

donde: $\quad U_{\mathrm{p}}$ es la uniformidad de distribución de presiones (\%)

$\mathrm{p}_{25}$ es la presión media del $25 \%$ de las localizaciones con una menor presión ( $\mathrm{kPa}$ ). Así, es la media de las 4 presiones más bajas de las 16 localizaciones

$\bar{p}$ es la presión media de todas las localizaciones muestreadas $(\mathrm{kPa})$, de forma que es la media de las 16 localizaciones $x$ es coeficiente de descarga de la ecuación característica del gotero

En el método de Burt (2004) la uniformidad de distribución de caudales se calcula según la ecuación:

$$
U D_{p}=1-\frac{1}{\sqrt{n}}\left(1-\text { media } \frac{q_{\text {min }}}{q_{\text {media }}}\right)
$$

donde: $\quad n$ es el número de emisores por planta, adoptándose el valor 1 ya que no había plantas en el experimento 
el término media $\frac{\mathrm{q}_{\text {min }}}{\mathrm{q}_{\text {media }}}$ se calcula con la ecuación (4)

media $\frac{\mathrm{q}_{\text {min }}}{\mathrm{q}_{\text {media }}}=\frac{\sum_{1}^{3} \frac{\mathrm{q}_{\text {min }}}{\mathrm{q}_{\text {media }}}}{3}$

donde:

\begin{abstract}
$\mathrm{q}_{\text {min }}$, para cada una de las tres localizaciones, es la media del $25 \%$ de los goteros con un caudal inferior, por lo que es la media de 407 caudales, según si la localización es al inicio y mitad o final del lateral, respectivamente

$q_{\text {media }}$, para cada una de las tres localizaciones, es la media de todos los caudales medidos. Así, es el caudal medio de 16 o 28 medidas, según si la localización es al inicio y mitad o final del lateral, respectivamente
\end{abstract}

La uniformidad de distribución de caudales obtenida con el método propuesto se obtuvo a partir de los registros proporcionados cada minuto por un sistema de supervisión y adquisición de datos (SCADA) instalado en la subunidad de riego (Duran-Ros et al., 2008) (Figura 3). Se utilizaron los datos de los transductores de presión DMP 331 P (DB Sensors, Thierstein, Alemania) con una precisión de medida de $0.175 \%$ y de los contadores volumétricos 405 S DN 15 (Sensus, Raleigh, EE.UU) con una precisión del 1\%, localizados al inicio, a $1 / 3$ y a $2 / 3$ de su longitud y a $7 \mathrm{~m}$ del final de cada lateral tal como se ha presentado en la Figura 1. Para poder comparar los resultados de $U D_{q}$ obtenidos con el método de evaluación propuesto con las $U_{\mathrm{q}}$ obtenidas con los métodos de evaluación de campo (Merriam y Keller (1978) y Burt (2004)), se utilizaron los datos recogidos por el SCADA durante los mismos instantes en los que se realizaron las evaluaciones de campo.

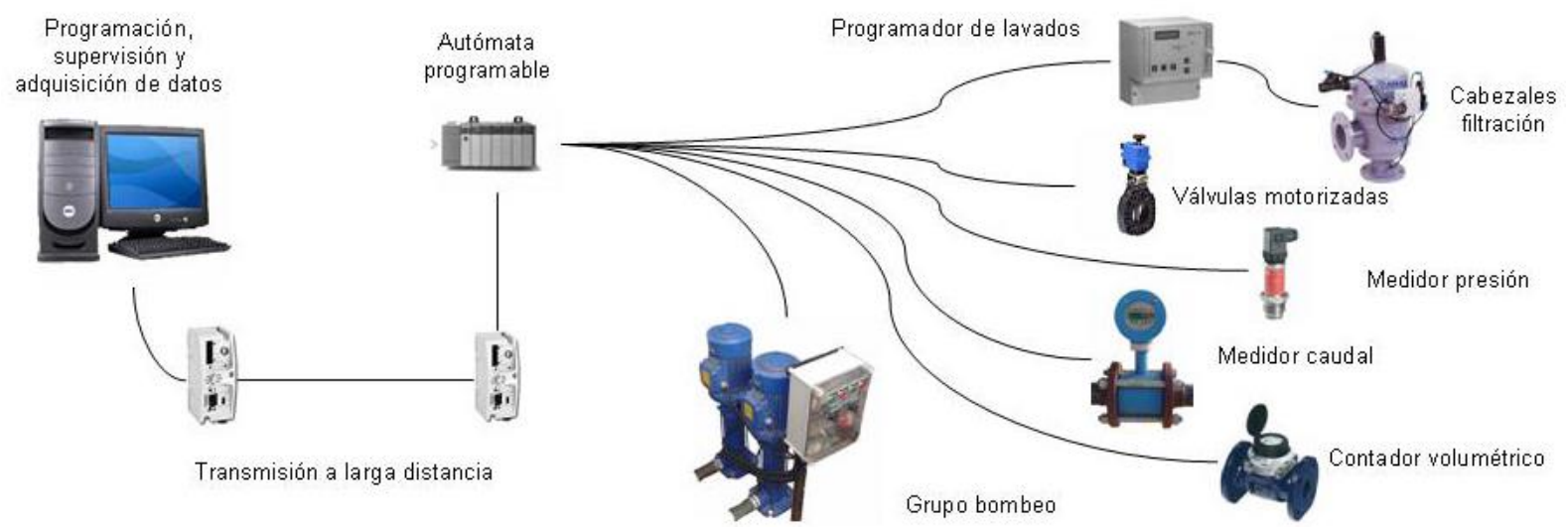

Figura 3. Esquema de los equipos que constituyen el sistema SCADA (sistema de supervisión, control, y adquisición de datos) implantado en la parcela experimental de riego.

A continuación se describe el procedimiento seguido para la determinación de la $U_{\mathrm{q}}$ con el nuevo método propuesto. Se seleccionaron los volúmenes registrados para los mismos tiempos en que se practicó la evaluación de campo, determinándose a partir de estos valores los caudales estimados para cada localización y lateral. Es preciso comentar que el caudal de las localizaciones inicial y $1 / 3$ y $2 / 3$ de la longitud del lateral representaba el caudal de todos los emisores del lateral desde el punto de medición hasta el final (¡Error! No se encuentra el origen de la referencia.). Por este motivo, para la estimación del 
caudal del tramo, se restó de estas localizaciones los caudales de los tramos posteriores según las ecuaciones (6), (7) y (8).

$$
\begin{aligned}
& q_{\text {final }}=Q_{\text {final }} \\
& q_{2 / 3}=Q_{2 / 3}-Q_{\text {final }} \\
& q_{1 / 3}=Q_{1 / 3}-Q_{2 / 3} \\
& q_{\text {inicio }}=Q_{\text {inicio }}-Q_{1 / 3}
\end{aligned}
$$

siendo:

$Q_{\text {inicio }}$ el caudal estimado en la localización inicio del lateral

$Q_{1 / 3}$ el caudal estimado en la localización $1 / 3$ de la longitud del lateral

$Q_{2 / 3}$ el caudal estimado en la localización 2/3 de la longitud del lateral

$Q_{\text {tinal }}$ el caudal estimado en la localización final del lateral

qinicio el caudal estimado en el tramo comprendido entre el inicio y la localización $1 / 3$ de la longitud del lateral

$q_{1 / 3}$ el caudal estimado en el tramo comprendido entre la localización 1/3 y 2/3 de la longitud del lateral

$\mathrm{q}_{2 / 3}$ el caudal estimado en el tramo comprendido entre la localización 2/3 de la longitud y el final del lateral

$q_{\text {final }}$ el caudal estimado en el tramo final del lateral

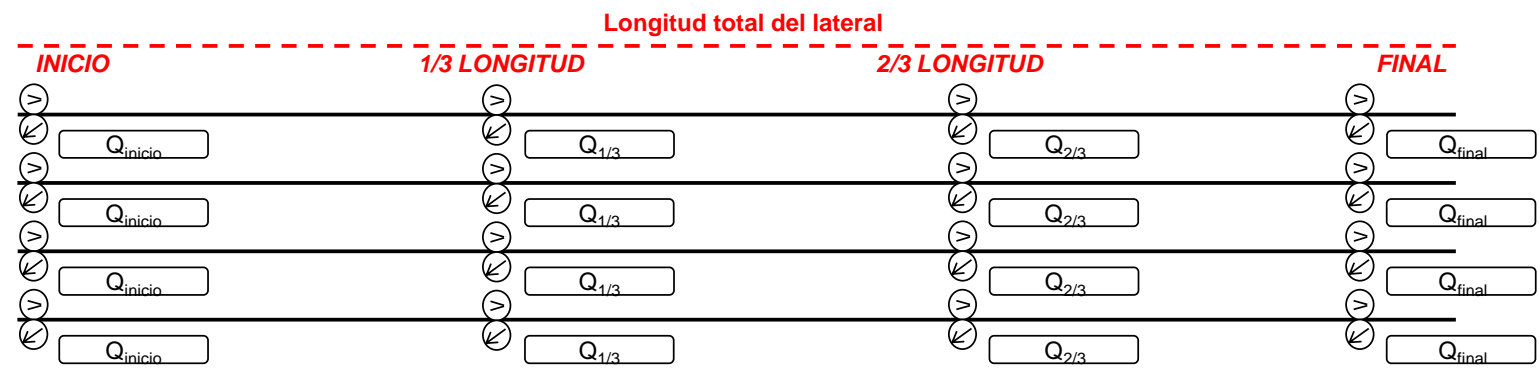

Leyenda:

$\ominus$ Medidor volumétrico (4) Transductor de presión

Figura 4. Localización de las mediciones con registro en continuo en los laterales de riego monitorizados.

Posteriormente, se dividió el caudal estimado para cada uno de los tramos por el número real de emisores del tramo, de manera que se obtuvo el caudal medio de los goteros para cada uno de los tramos del lateral. Finalmente, utilizando la ecuación (1), se obtuvo un valor de la $U_{\mathrm{q}}$ relacionando la media del $25 \%$ de los emisores con menores caudales estimados y la media de los caudales estimados. 


\section{3- Resultados y discusión}

La $U_{\mathrm{p}}$ estuvo siempre por encima del $97 \%$ y con un valor promedio para todas las medidas de $98.3 \%$, lo que es indicativo de que el sistema estaba correctamente diseñado y operaba adecuadamente.

2.

Los resultados de la $U D_{q}$ para las 13 evaluaciones realizadas se indican en la tabla

Tabla 1. Comparación entre las $U D_{q}(\%)$ obtenidos con los distintos métodos de evaluación.

\begin{tabular}{|c|c|c|c|}
\hline Evaluación & Merriam y Keller (1978) & Burt (2004) & Método propuesto \\
\hline 1 & 96,30 & 94,24 & 89,13 \\
\hline 2 & 81,31 & 71,84 & 49,39 \\
\hline 3 & 90,91 & 86,06 & 66,38 \\
\hline 4 & 71,81 & 62,49 & 74,55 \\
\hline 5 & 75,16 & 58,85 & 60,73 \\
\hline 6 & 68,41 & 58,25 & 61,48 \\
\hline 7 & 95,50 & 95,78 & 90,80 \\
\hline 8 & 71,01 & 50,71 & 38,76 \\
\hline 9 & 69,96 & 58,37 & 36,38 \\
\hline 10 & 70,59 & 58,20 & 70,60 \\
\hline 11 & 12,90 & 58,41 & 55,80 \\
\hline 12 & 95,09 & 91,07 & 78,66 \\
\hline 13 & 88,25 & 66,12 & 83,34 \\
\hline
\end{tabular}

Los valores de la $U_{\mathrm{q}}$ obtenidos aplicando cada uno de los métodos han resultado distintos. Se atribuye esta discrepancia a que tanto el método de Merriam y Keller (1978) como el de Burt (2004) sólo miden el caudal de algunos emisores, mientras que el método propuesto determina, de forma indirecta, el caudal de todos los goteros de la instalación. Esto concuerda con Juana et al. (2007) quienes cuestionaron si con la selección de las localizaciones en el método de Merriam y Keller (1978), se podía asumir la media de caudales muestreados como la media de caudales de toda la unidad de riego. A partir de los valores obtenidos con el método propuesto no se puede inferir el valor de la UDq que se obtendría utilizando el método de Merriam y Keller (1978) ni el de Burt (2004) ya que el coeficiente de correlación fue de 0.24 y 0.48 respectivamente, siendo ambos valores muy bajos. Sin embargo, se obtuvo una buena correlación entre los valores obtenidos con los métodos de Merriam y Keller (1978) y Burt (2004) $\left(R^{2}=0.87\right)$, explicable por las semejanzas entre los métodos por lo que se refiere a la localización de los goteros que se muestrean.

En la Figura 5 se presenta la evolución de la $U D_{q}$ en función de las horas de riego acumuladas para cada método de medición, 


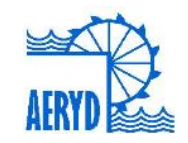

XXXIII Congreso Nacional de Riegos

Universitat Politècnica de València, Valencia 2015

DOI:http://dx.doi.org/10.4995/CNRiegos.2015.1526

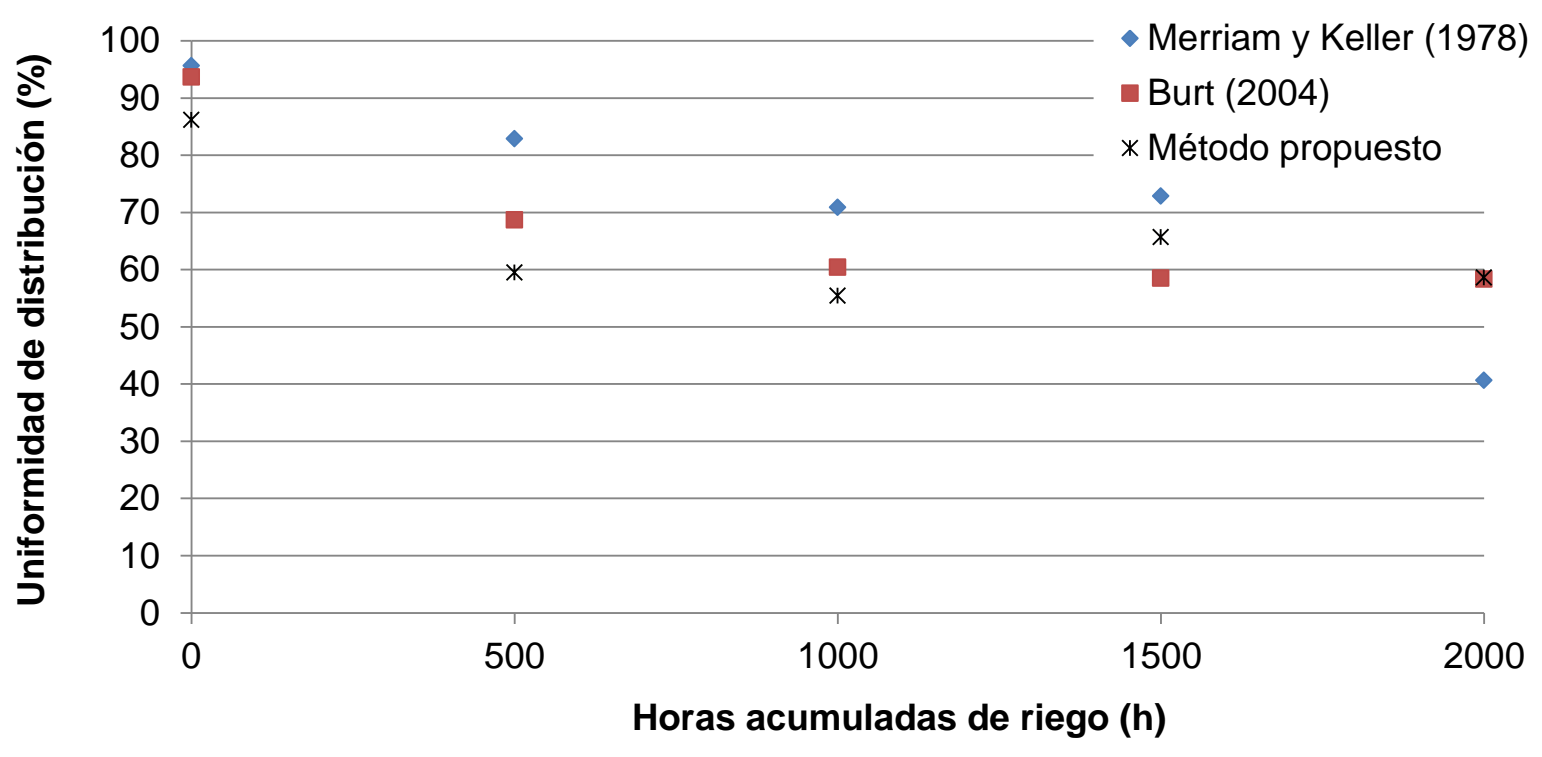

Figura 5 . Evolución de la $U D_{q}$ en función de las horas de riego y el método de evaluación utilizado.

El método de Merriam y Keller (1978) penaliza en gran medida los emisores completamente obturados del final del lateral ya que utiliza los dos últimos emisores del mismo. Diversos autores han constatado que es más probable encontrar emisores completamente obturados al final de los laterales de riego, especialmente cuando se utilizan aguas regeneradas (Duran-Ros et al., 2009; Ravina et al. 1992, 1997; Trooien et al. 2000) debido a que con el menor caudal que circula por esta zona hay una mayor cantidad de deposiciones (Ravina et al. 1992, Trooien et al. 2000; Hills et al. 2000). En las evaluaciones en las que se obtuvo menor UD $_{\mathrm{q}}$ según el método de Merriam y Keller (1978) se registraron los mayores números de emisores completamente obturados, lo que se observa (Figura 5) con una caída de la $U D_{\mathrm{q}}$ hasta el $40 \%$ que se produce al llegar a las $2000 \mathrm{~h}$ de funcionamiento. El método de Burt (2004) no penaliza tanto sobre el valor de la UD por la presencia de goteros completamente obturados del final del lateral, ya que en la localización del final del lateral se miden 7 emisores por lateral y no únicamente los 2 últimos.

El método propuesto, presenta (Figura 5) unos valores parecidos a los determinados con el método de Burt (2004) pero con la ventaja que se evita la realización de las medidas discretas y manuales en campo de caudal y presión. El método propuesto permite además, obtener valores de $U D_{q}$ de sistemas de riego por goteo enterrado sin tener que desenterrar los laterales de riego. Sin embargo, la nueva metodología presentada requiere una mayor tecnificación de la instalación, con la implementación de medidores de volumen y presión en las localizaciones estratégicas y equipos para registrar los datos. Con este sistema se puede controlar en tiempo real y continuo el funcionamiento de las instalaciones de riego localizado. La metodología propuesta se enmarca dentro de las nuevas tecnologías del riego que permiten alcanzar mayores eficiencias en la utilización del agua (Ruíz-Canales y Ferrández-Villena, 2015).

\section{7- Conclusiones y recomendaciones}

Para la determinación de la $U_{\mathrm{q}}$ se han comparado los métodos de Merriam y Keller (1978) y de Burt (2004) con un nuevo procedimiento desarrollado a partir del de Merriam y Keller midiendo el caudal y la presión en distintos puntos del lateral de riego en lugar de en determinados goteros. Existe una evolución temporal parecida de las $\mathrm{UD}_{\mathrm{q}}$ con los tres 
métodos, pero la presencia de obturaciones tiene mucha incidencia, especialmente en el método de Merriam y Keller (1978) puesto que solamente utiliza 2 goteros del final del lateral y es muy probable que se obturen después de largos períodos de utilización con aguas con potencial de atascamiento.

Los resultados indican la gran incidencia que tiene la obturación de los goteros en los valores de la $U D_{\mathrm{q}}$. El método propuesto presenta como ventajas su automatización y que considera indirectamente todos los goteros del sistema, de forma que no se resulta tan afectado por la obturación de los goteros evaluados como sucede con el método de Merriam y Keller (1978), en mayor medida, y el de Burt (2004) y presenta también la posibilidad de evaluar instalaciones de riego por goteo enterrado que serían imposibles de evaluar sin desenterrar los laterales de riego.

\section{8.- Agradecimientos}

Los autores quieren agradecer al Ministerio de Economía y Competitividad la concesión de la ayuda para la realización del proyecto de investigación referencia CGL201231180. También agradecen a Regaber y al Ayuntamiento de Celrà (Girona) su ayuda en el desarrollo de las experiencias.

\section{9- Bibliografía}

ASAE, 1998. ASAE Standards. ASAE, 2950 Niles Road, St Joseph, MI 49085-9659, USA.

Barragán, J., Bralts, V. \& Wu, I.P (2006). Assessment of Emission Uniformity for Microirrigation Design. Biosystems Engineering, 93 (1), 89-97.

Bucks, D. A., Nakayama, F. S. \& Gilbert, R. G. (1979). Trickle irrigation water quality and preventive maintenance. Agricultural Water Management, 2 (2), 149-162.

Burt, C. (2004). Rapid field evaluation of drip and microspray distribution uniformity. Irrigation and Drainage Systems, 18, 275-297.

Duran-Ros, M., Puig-Bargués, J., Arbat, G., Barragán, J. \& Ramírez de Cartagena, F. (2008). Definition of a SCADA system for a microirrigation network with effluents. Computers and Electronics in Agriculture, 64 (2), 338-342.

Duran-Ros, M., Puig-Bargués, J., Arbat, G., Barragán, J. \& Ramírez de Cartagena, F. (2009). Effect of filter, emitter and location on clogging when using effluents. Agricultural Water Management, 96 (1), 67-79.

Hills, D.J., Tajrishy, M.A. \& Tchobanoglous, G. (2000). The influence of filtration on ultraviolet disinfection of secondary efluent for microirrigation. Transactions of the ASAE 43 (6), $1499-1505$.

Juana, L., Rodriguez-Sinobas, L., Sánchez, R. \& Losada, A. (2007). Evaluation of drip irrigation: Selection of emitters and hydraulic characterization of trapezoïdal units. Agricultural Water Management, 90, 13-26.

Merriam, J. L. \& Keller, J. (1978). Farm irrigation evaluation: A guide for management. Department of Agricultural and Irrigation Engineering, Utah State University, Logan, Utah. 
Ravina, I., Paz, E., Sofer, Z., Marcu, A., Shisha, A. \& Sagi, G. (1992). Control of emitter clogging in drip irrigation with reclaimed wastewater. Irrigation Science. 13 (3) , 129139.

Ravina, I., Paz, E., Sofer, Z., Marcu, A., Shisha, A., Sagi, G., Yechialy, Z. \& Lev, Y. (1997). Control of clogging in drip irrigation with stored treated municipal sewage effluent. Agricultural Water Management, 33 (2-3), 127-137

Ruiz-Canales, A. \& Ferrández-Villena, M. (2015) New proposals in the Automation and remote control of wàter management in agriculture: Agromotic Systems. Agricultural Water Management, 151, 1-3.

Trooien, T.P., Lamm, F.R., Stone, L.R., Alam, M., Rogers, D.H., Clark, G.A. \& Schlegel, A.J., (2000). Subsurface drip irrigation using livestock wastewater: dripline flow rates. Applied Engineering. Agriculture, 16 (5), 505-508.

Vermeiren, L. \& Jobling, G. A. (1986). Riego localizado. Riego y Drenaje, 36. FAO, Roma.

Wu, I-P., Barragán, J. \& Bralts, V. (2007). Field performance and evaluation. En: Lamm, F.R., Ayars, J.E. \& Nakayama, F.S. (Eds.) Microirrigation for crop production. Design, Operation, and Management (357-388). Amsterdam. Elsevier. 